\title{
Transatlantica
}

Revue d'études américaines. American Studies Journal

\section{La rivalité Paris/New York à travers les expositions récentes d'œuvres françaises et américaines.}

Quelques remarques.

\section{Sophie Le Cam}

\section{(2) OpenEdition}

\section{Journals}

Édition électronique

URL : http://journals.openedition.org/transatlantica/553

DOI : $10.4000 /$ transatlantica.553

ISSN : 1765-2766

Éditeur

AFEA

Référence électronique

Sophie Le Cam, «La rivalité Paris/New York à travers les expositions récentes d'œuvres françaises et américaines. », Transatlantica [En ligne], 1 | 2003, mis en ligne le 24 mars 2006, consulté le 29 avril 2021. URL : http://journals.openedition.org/transatlantica/553 ; DOI : https://doi.org/10.4000/ transatlantica.553

Ce document a été généré automatiquement le 29 avril 2021

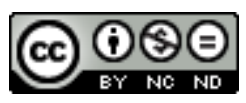

Transatlantica - Revue d'études américaines est mis à disposition selon les termes de la licence Creative Commons Attribution - Pas d'Utilisation Commerciale - Pas de Modification 4.0 International. 


\title{
La rivalité Paris/New York à travers les expositions récentes d'œuvres françaises et américaines.
}

Quelques remarques.

\author{
Sophie Le Cam
}

1 L'attribution en 1964 du Grand Prix de la 32ème Biennale de Venise à l'artiste américain Robert Rauschenberg a constitué un tournant dans les relations artistiques entre la France et les États-Unis. Elle a entériné le nouveau rôle de New York en tant que centre de l'art moderne en lieu et place de Paris déclenchant de nombreuses polémiques entre les protagonistes du monde de l'art français et américain. La « rivalité » entre les deux univers artistiques a alors atteint un point culminant.

2 Au cours de l'année 2002, le Musée Maillol à Paris a abrité une exposition consacrée aux œuvres récentes de Robert Rauschenberg (Fondation Dina Vierny - Musée Maillol, du 6 juin au 14 octobre 2002). Cette dernière s'ouvrait sur une présentation de l'artiste remettant en cause sa victoire de l'époque. Il aurait, selon le terme employé par les organisateurs, "usurpé» le Grand Prix à l'artiste français François Bissière. La remarque faite à l'occasion de cette exposition témoigne de la persistance avec laquelle certains protagonistes du monde de l'art n'ont cessé, jusqu'à aujourd'hui, d'envisager les rapports entre Paris et New York sous un angle conflictuel. Si cet aspect est souvent ignoré, la "rivalité » entre les deux métropoles reste en effet vivace, malgré les dénégations, sincères ou intéressées, des acteurs concernés. Les analyses relatives aux tensions franco-américaines dans le domaine artistique portent en majorité sur des faits antérieurs, les éventuels antagonismes persistant actuellement faisant l'objet de brèves allusions. Le rappel de ces clivages contribue pourtant en soi à entretenir une certaine défiance entre les deux univers. Ce phénomène est notamment perceptible à travers les expositions présentant côte à côte des œuvres d'artistes français et américains.

3 Les expositions soulignant les similitudes et différences entre œuvres américaines et françaises appartenant à des courants non contemporains entendent le plus souvent 
démontrer quel groupe d'artistes, américain ou français, a eu la primauté d'influencer l'autre et de marquer l'histoire de l'art en général. C'est au début des années 50 que ce procédé est apparu pour la première fois dans un esprit de confrontation. Les polémiques entourant l'exposition Young Painters from US and France organisée en 1950 par Leo Castelli à la galerie Sidney Janis sont caractéristiques. Une quinzaine de rapprochements furent alors établis entre peintres de l'École de Paris et peintres de l'École de New York, en particulier entre les œuvres de Pierre Soulages et Franz Kline, comparaisons qui continuent actuellement à faire l'objet de multiples analyses, voire de débats. Depuis lors, de nombreuses expositions ont offert la possibilité de comparer l'évolution de la création artistique de part et d'autre de l'Atlantique, selon des approches et à des fins variées. Depuis la seconde moitié des années 90 le phénomène a cependant pris une nouvelle dimension. À une époque où les rapprochements entre les univers artistiques français et américains croient en nombre et en ampleur - une structure comme FRAME est là pour en témoigner - et où l'intérêt respectif des publics américains et français pour toutes les formes et toutes les périodes de l'art existant outre-Atlantique se développe - François Brunet en a traité un de ces aspects dans cette même revue (Transatlantica 2) sous le titre «L'art américain d'avant Pollock est-il à la mode ?» - il est d'autant plus surprenant que les parallèles effectués entre artistes français et américains soient en réalité autant de prétextes à les opposer.

L'exposition De Klein à Warhol: face-à-face France/États-Unis présentée au Musée d'Art moderne et d'art contemporain de Nice au cours de l'hiver 1997-98 et qui a rassemblé des œuvres françaises et américaines du Centre Georges Pompidou et du Musée d'Art moderne et d'art contemporain de Nice illustre bien l'approche ambivalente de ces événements actuellement en voie de multiplication. Comme il est dit en introduction du catalogue accompagnant l'exposition, l'objet essentiel de cette dernière est d'évoquer «les échanges artistiques entre la France et les États-Unis autour du Nouveau Réalisme et du Pop Art ", et ce, dès le début des années 50 où se forme le groupe français. Le ton adopté par les organisateurs de l'exposition et rédacteurs du catalogue, Sophie Duplaix et Gilbert Perlain, trahit cependant une volonté de réaffirmer l'originalité de la création artistique française de l'époque et de réagir aux propos antérieurs de la critique américaine, selon eux chauvins : «Bien que l'apport français, dans la diversité de ses démarches, apparaisse particulièrement novateur, il semble qu'une grande partie de la critique américaine n'ait pas été disposée à en reconnaître l'originalité. Ainsi Lucy Lippard qualifie de "laborieuse et fortement surréaliste » la participation en 1962 des Nouveaux Réalistes à l'une des premières expositions new-yorkaises où ils figurent en bonne place aux côtés des Américains, à la Sidney Janis Gallery; dans le même esprit, Irving Sandler affirme, de façon plus générale, que les Nouveaux Réalistes «n'ont pas particulièrement impressionné les milieux new-yorkais ». Il convient de conserver toutefois une certaine distance vis-à-vis d'un phénomène qui, en définitive, n'est pas propre à cette période, mais traverse l'histoire des rapports entre la France et les États-Unis depuis l'après-guerre." (13) Cette dernière remarque suggère que les relations artistiques entre les deux pays demeurent entachées de rivalités, et afin de justifier de la crédibilité de leur propos, Sophie Duplaix et Gilbert Perlain incluent dans le catalogue un entretien avec Pontus Hulten, de nationalité suédoise et témoin privilégié des développements artistiques de l'époque. Ils font ainsi une allusion plus directe à la motivation profonde de cette exposition: dénoncer le nationalisme excessif de certains Américains à travers « (...) une lecture nuancée d'une époque où domine essentiellement, côté américain, une 
critique triomphaliste ». (13) Il apparaît que c'est l'interprétation qui est donnée de ces expositions qui, pour une grande part, transforme ces mises en apposition d'œuvres en confrontations.

5 C'est un constat récurrent sur lequel nous reviendrons à propos d'expositions plus récentes. Ainsi, si certains observateurs tel que Robert Rosenblum ont vu à travers l'exposition De Klein à Warhol: face-à-face France/États-Unis un dialogue entre artistes français et américains établi dans un esprit d'échanges amicaux, plus nombreux ont été les commentaires des critiques cautionnant l'idée d'une confrontation perdurant dans le domaine artistique entre la France et les États-Unis. Même si cette volonté de rétablir la valeur des artistes français, trop souvent dévalorisés par la critique américaine selon ces derniers, concerne une époque révolue, il est un fait qu'elle constitue un aspect des relations actuelles. Dans un article intitulé "Nice et Beaubourg: un pont sur l'Atlantique », le critique journaliste Damien Sausset insiste sur le fait que le principal apport de cette exposition est de donner une vision nouvelle de l'importance réelle de la création des Nouveaux Réalistes : «(...) la réussite de cette exposition réside dans sa volonté de démontrer combien les œuvres d'Arman, de Raysse, de César, de Dufrêne, de Hains et de Klein témoignent d'une résistance à la représentation figurative qui anime alors les œuvres américaines. Le poids excessif de l'art américain de ce début des années 60 se trouve soudain relativisé, comme oblitéré par la capacité des artistes français à ouvrir leurs œuvres au regard subjectif des spectateurs ». (27) Il donne un exemple concret de l'effet obtenu par la mise en présence de ces œuvres françaises et américaines des années 60. L'exposition face-à-face de l'œuvre de Claes Oldenburg Cemetery Flag (1960) et de Jacques de la Villeglé les Affiches Lacérées (1950) permet selon lui de mesurer « les écarts qui existent entre ces Nouveaux Réalistes qui n’hésitent pas à tenter sans grand succès, de conquérir le marché américain et des artistes new-yorkais qui, souvent, jugent la production française trop sentimentale ».

6 Loin de s'estomper, ce phénomène a donc pris une dimension supplémentaire en ce début de XXIème siècle avec des expositions d'envergure internationale telles que Les Années Pop 1956-1968 et Paris Capitale des Arts 1900-1960. La présentation d'œuvres d'origines diverses a permis d'étendre les analyses comparatives aux artistes européens afin de mieux justifier des rectifications entamées à l'égard des artistes français.

7 Présentée au Centre Georges Pompidou du 15 mars au 18 juin 2001, Les Années Pop 1956-1968 revient par ce biais sur les tensions liées à l'émergence des artistes du groupe des Nouveaux Réalistes et du Pop Art. Rédigé par Jean-Jacques Aillagon, alors Président du Centre Georges Pompidou, l'avant-propos qui ouvre le catalogue de l'exposition n'est d'aucune ambiguïté à cet égard. L'un des buts essentiels de la manifestation est de rappeler l'importance du rôle joué par les artistes européens, et en particulier français, dans l'émergence du mouvement Pop Art. À travers un essai intitulé «Nouveaux Réalismes et Pop Art ", Catherine Grenier, commissaire générale de l'exposition, souligne l'apport essentiel, voire fondateur, des Nouveaux Réalistes au mouvement Pop Art. Sans exclure la participation des artistes américains au développement du groupe des Nouveaux Réalistes dans les années 50, Catherine Grenier entend démontrer que ce mouvement est bien antérieur au mouvement Pop Art apparu dans les années 60. Elle étaye son argumentation en précisant comment de nombreux artistes américains, tels que Robert Rauschenberg et Jasper Johns ont utilisé des techniques créées par les artistes français. Aussi radicale soit elle, la perspective voulue par Catherine Grenier n'est pas aussi marginale qu'elle pourrait le sembler aux yeux de certains spécialistes. 
8 Alain Jacquet, artiste français ayant évolué sur la scène artistique de l'époque tient des propos similaires. Tout en évoquant l'intensité et la richesse des échanges entretenus par les artistes français et américains, il insiste sur la nécessité de «...remettre les pendules à l'heure» et de démontrer que le Pop Art n'est pas «...un phénomène exclusivement américain ». Il estime par ailleurs important de prendre en compte les effets de la politique étrangère américaine de l'époque qui visait à augmenter l'influence des États-Unis en Europe par des voies culturelles et artistiques. À la lumière de cet exemple, il apparaît que des personnalités ayant eu une expérience concrète de ces événements y demeurent sensibles, exprimant leur volonté de revenir sur des aspects litigieux. Ils participent ainsi à faire perdurer un débat commencé de longue date.

9 Autre exposition récente ayant contribué à rappeler les nombreux clivages ponctuant les relations artistiques franco-américaines, et sur lequel il convient donc de s'arrêter, Paris Capitale des Arts 1900-1960 (Royal Academy of Arts, Londres, du 26 janvier au 19 avril 2002 et Guggenheim Museum, Bilbao, du 21 mai au 3 septembre 2002). La dimension internationale de celle-ci est d'autant plus marquée qu'elle n'a pas été organisée sur une initiative française ou américaine. Présentée successivement à Londres et à Bilbao dans le courant de l'année 2002, elle témoigne d'une généralisation de l'intérêt soulevé par cet aspect des rapports franco-américains. Sarah Wilson, l'un des commissaires principaux de l'exposition, analyse à plusieurs reprises les tensions apparues entre les protagonistes français et américains au cours des années 60 . Figurait par ailleurs dans le catalogue de l'exposition un essai rédigé par Eric de Chassey dont le titre «Paris-New York : rivalités et dénégations » est, à lui seul, révélateur de la perspective dans laquelle l'exposition a été conçue. Son évocation des rapports conflictuels entretenus par les acteurs des scènes artistiques française et américaine depuis l'après-guerre jusqu'à la fin des années 60 se conclut sur cette remarque à propos de l'état actuel des relations franco-américaines : «[À la fin des années 60] alors que les expositions consacrées aux artistes américains se multipliaient à Paris, les artistes français éprouvaient des difficultés à exposer leurs œuvres aux États-Unis. D’emblée, leur art était considéré comme 'provincial' : trop français. Un préjugé qu'il faut encore assumer ». (350) Une nouvelle fois il est suggéré que les rivalités passées demeurent inscrites dans les esprits.

10 À défaut d'un réel sentiment d'animosité, il subsiste sans aucun doute une grande sensibilité à l'égard de ce qui est décrit comme le déplacement du centre de l'activité artistique mondiale de Paris vers New York. Malgré son caractère antérieur, c'est sans doute parce qu'elle continue à avoir des conséquences sur la nature des relations actuelles que cette question conserve un caractère contemporain. Une autre raison justifiant la pérennité de cette rivalité est la multiplication de ses implications. À l'image des travaux de Serge Guilbaut ou de l'Américaine Laurie J. Monahan, ces expositions ont été l'occasion d'évoquer, voire de mettre en exergue comme l'ont notamment fait les organisateurs de Paris Capitale des Arts 1900-1968 les enjeux politiques sous-jacents au contexte artistique. S'il est quasiment indéniable qu'assurer la prééminence artistique de New York a été l'un des moyens utilisés à l'époque par le gouvernement américain pour s'élever contre l'expansion du communisme en Europe, il est difficile d'évaluer le rôle - s'il existe - attribué de nos jours par les politiques à l'art dans la défense des intérêts nationaux. C'est en effet aujourd'hui avant tout vers 
les sphères financières et économiques que l'essentiel des rapports concurrentiels se sont déplacés.

11 Si pendant très longtemps, les acteurs français, qu'ils soient artistes, marchands ou responsables officiels, ont dans leur majorité ignoré l'importance des enjeux économiques, depuis la fin des années 90 ils semblent avoir progressivement pris en compte la nécessité dans laquelle ils se trouvaient de s'imposer face aux États-Unis sur le terrain commercial. Depuis l'exposition Les Années Pop 1956-1968 au début de l'année 2001, beaucoup d'observateurs ont mis en exergue le clivage existant entre les artistes français et leurs homologues américains, non seulement en terme de notoriété mais aussi de cote sur le marché. Même si les comparaisons effectuées mettent surtout en lumière le "fossé » qui existe entre la valeur monétaire des œuvres des artistes des deux nations, elles sont néanmoins une preuve de la nouvelle approche des protagonistes français à l'encontre de la vraie valeur des œuvres sur le marché. Suite à l'engouement, bien que limité au contexte parisien, suscité par la vente «Manifeste pour la Nouvelle Figuration » organisée le 4 février 2002 par les études Poulain-Le Fur et Robin Fattori, cette volonté de se mesurer sur un terrain jusque là négligé s'est affirmée. Si, à cette occasion, la plus haute enchère a été atteinte avec une toile d'Andy Warhol, les œuvres des artistes Pop américains n'ont généralement pas emporté un succès à la mesure de celui habituellement connu, et c'est surtout l'élévation de la cote des artistes français qui aura été retenue. C'est donc d'un point de vue avant tout économique que la rivalité artistique entre Paris et New York - si tant est qu'elle subsiste - est appelée à évoluer de manière significative, et ce d'autant plus depuis la disparition du monopole des commissaires-priseurs français. Avec l'ouverture du marché français aux maisons anglo-saxonnes, professionnels français et américains sont en effet directement confrontés, donnant une nouvelle dimension à la « rivalité » artistique entre les deux métropoles.

\section{BIBLIOGRAPHIE}

American Artists in the American Ambassador's Residence in Paris, Paris, Jeanne Greenberg Art Advisory, 1998.

Les Années Pop 1956-1968, Paris, Centre Georges Pompidou, 2001.

Breerette, Geneviève, « Une exposition inédite sur la naissance d'une culture populaire », Le Monde, vendredi 16 mars 2001.

Dagen, Philippe « Alain Jacquet, peintre : « le pop n'est pas un phénomène exclusivement américain », Le Monde, vendredi 16 mars 2001.

De Klein à Wahrol :face-à-face France/Etats-Unis, Paris, Réunion des Musées Nationaux, 1998.

Grenier, Catherine, propos recueillis par Geneviève Breerette, « Le Centre Pompidou explore sous toutes ses formes l'art des ‘Golden Sixties’ », Le Monde, vendredi 16 mars 2001. 
Guilbaut, Serge, Comment New York vola l'idée d'art moderne ?, Nîmes, Editions Jacqueline Chambon, 1988.

Paris Capitale des Arts 1900-1960, Londres, Royal Academy of Arts ; Hazan pour l'édition française, 2002.

Reconstructing Modernism Art in New York, Paris, and Montreal 1945-1964, édité par Serge Guilbaut, Cambridge, Mass., MIT Press, 1990.

Rosenblum, Robert, « Pop Art Revisited », Art Forum, V39, N5, janvier 2001, 37.

Sandler, Irving, The Triumph of American Painting, New York, Harper Collins, 1970, 1977.

Sausset, Damien, « Nice et Beaubourg : un pont sur l'Atlantique », L’Eil, n492, janvier 1998, 27.

INDEX

Thèmes : Trans'Arts 